\title{
Clinical and molecular consequences of exon 78 deletion in $D M D$ gene
}

\author{
Monica Traverso $^{1} \cdot$ Stefania Assereto $^{2} \cdot$ Serena Baratto $^{2} \cdot$ Michele lacomino $^{1} \cdot$ Marina Pedemonte $^{2}$. \\ Maria Cristina Diana ${ }^{2} \cdot$ Marta Ferretti $^{2} \cdot$ Paolo Broda $^{2} \cdot$ Carlo Minetti $^{1,2} \cdot$ Elisabetta Gazzerro $^{2}$ - Francesca Madia ${ }^{3}$. \\ Claudio Bruno ${ }^{2} \cdot$ Federico Zara $^{3} \cdot$ Chiara Fiorillo $^{1,2}$
}

Received: 5 October 2017 / Revised: 5 February 2018 / Accepted: 19 February 2018

(c) The Author(s) under exclusive licence to The Japan Society of Human Genetics 2018

\begin{abstract}
We present a 13-year-old patient with persistent increase of serum Creatine Kinase (CK) and myalgia after exertion. Skeletal muscle biopsy showed marked reduction of dystrophin expression leading to genetic analysis of DMD gene by MLPA, which detected a single deletion of exon 78 . To the best of our knowledge, $D M D$ exon 78 deletion has never been described in literature and, according to prediction, it should lead to loss of reading frame in the dystrophin gene. To further assess the actual effect of exon 78 deletion, we analysed cDNA from muscle mRNA. This analysis confirmed the absence of $32 \mathrm{bp}$ of exon 78. Exclusion of exon 78 changes the open reading frame of exon 79 and generate a downstream stop codon, producing a dystrophin protein of 3703 amino acids instead of 3685 amino acids. Albeit loss of reading frame usually leads to protein degradation and severe phenotype, in this case, we demonstrated that deletion of DMD exon 78 can be associated with a functional protein able to bind DGC complex and a very mild phenotype. This study adds a novel deletion in $D M D$ gene in human and helps to define the compliance between maintaining/disrupting the reading frame and clinical form of the disease.
\end{abstract}

\section{Introduction}

Duchenne muscular dystrophy (DMD, OMIM \#310200) and its milder allelic variant and Becker muscular dystrophy (BMD, OMIM \#300376) are both caused by mutations in the $D M D$ gene located on Xp21. DMD represents the most common genetic neuromuscular disease of childhood, with an incidence of 1 in 3500 male live birth [1].

DMD is characterized by progressive muscle degeneration, with overt onset of muscle weakness by age 7-8 years, subsequent loss of mobility and premature death. The progression of BMD is slower and signs and symptoms appear usually after 10 years of age. In many BMD patients, mobility is preserved for a long time.

Chiara Fiorillo

chiara.fiorillo@edu.unige.it

DINOGMI, University of Genoa, Genoa, Italy

2 Paediatric Neurology and Muscular Diseases Unit, University of Genoa and G. Gaslini Institute, Genoa, Italy

3 Laboratory of Neurogenetic and Neuroscience, G. Gaslini Institute, Genoa, Italy
The $D M D$, one of the largest human gene, consists of 79 exons, encodes a 14-kb mRNA (NM_004006.2) [2-4] and produces the dystrophin. Dystrophin is a sarcolemmaassociated protein, which binds cytoskeletal actin through its N-terminal domain, and a complex of dystrophinassociated proteins through its C-terminal domain [3].

Intragenic deletions and duplications account for over two-thirds of the mutations leading to DMD and BMD and the remainder may be due to point mutations $[5,6]$.

The major determinant of disease severity is whether the mutation results in an mRNA that maintains an open reading frame (ORF) allowing translation of a functional amino- and carboxy-terminal [7].

In Duchenne patients, mutations induce a shift in the reading frame leading to prematurely truncated, dysfunctional dystrophin. In Becker patients, in-frame mutations allow the synthesis of internally deleted, but largely functional dystrophin [8].

In this study, we provide a clinical and histopathological portrait of previously unreported $D M D$ exon 78 deletion, particularly focusing on genotype-phenotype correlations and molecular effect of this mutation that appears to be exception to the reading-frame rule. 

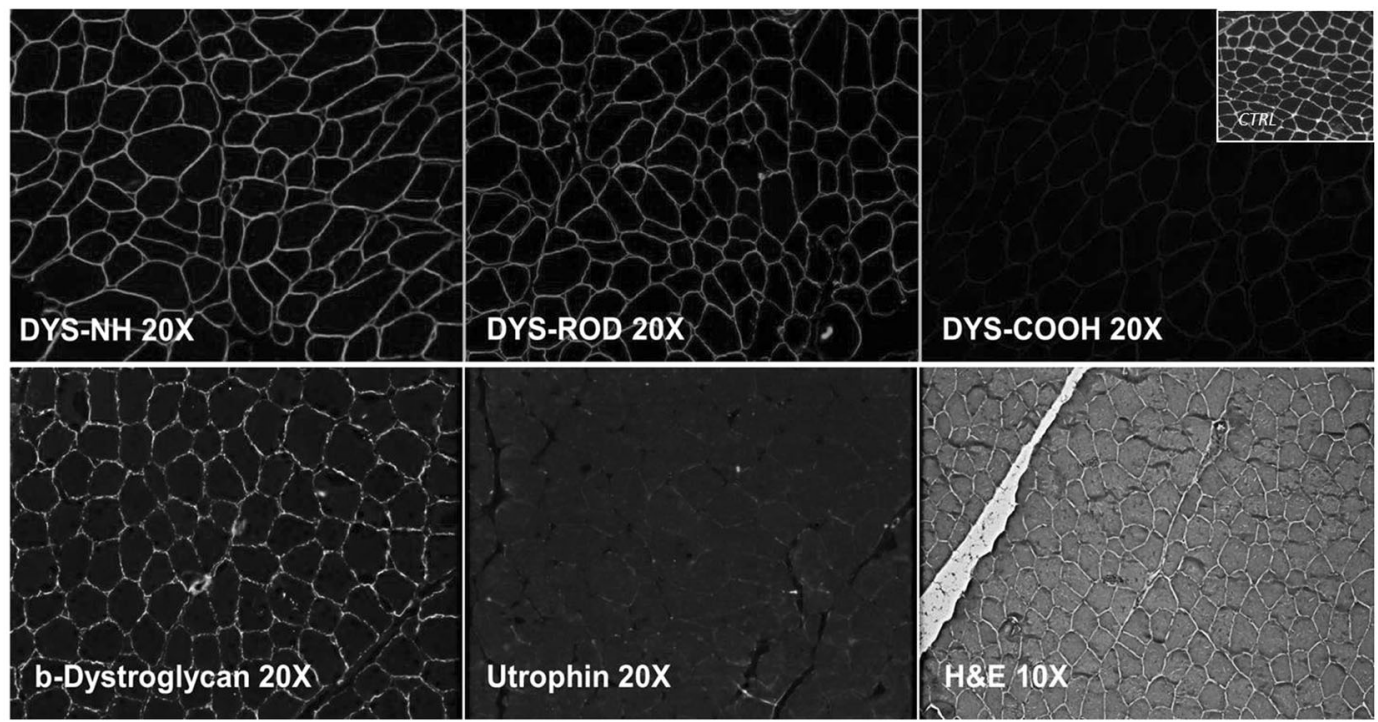

Fig. 1 Upper panel: frozen muscle tissue sections of the patient immunolabelled with Novocastra antibodies against dystrophin Nterminal (DYS-NH), dystrophin Rod domain (DYS-ROD) and dystrophin C-terminal (DYS-COOH) with control muscle section (CTRL) in square box in upper corner. Final magnification, $\times 20$. In lower

\section{Case description}

A 13-year-old boy came to our unit for persistent increase of serum Creatine Kinase (CK), associated with myalgia after exertion.

Born at term from healthy unrelated parents, the boy displayed normal psychomotor development. Clinical history was unremarkable until age 12 years when he started agonist boating with training six times a week. Motor performance were excellent and alike his peers, however, he started complaining of myalgia after exercise. For persistence of the symptoms, routine lab exams were indicated by general paediatrician at age 12 years and 5 months, which revealed increased CK level (2465 U/L), whereas other results were all normal. After a period of rest from exercise, CK levels were measured again at 1 and 2 months later and resulted still elevated (285 U/L and $633 \mathrm{U} / \mathrm{L}$, respectively). Being in good health, he resumed boating, but at age 12 years and 9 months, he was admitted in Emergency Department for severe pain in chest and interscapular region. Electrocardiogram documented unspecific alteration of right bundle transmission. Cardiological examination was clinically normal. Troponin was normal, however CK was still elevated (751 U/L).

At age 13 years, he was admitted to our hospital. CK at admission was $680 \mathrm{U} / \mathrm{L}$. Height and weight were above 70th percentile. Intellectual functions were normal. Neurological examination revealed only mild hypotrophy of shoulder girdle and pectoral muscles. Strength was normal. panel: frozen muscle tissue sections of the patient immunolabelled with Novocastra antibodies against beta-dystroglycan and utrophin (final magnification, $\times 20$ ) and stained with standard $\mathrm{H} \& \mathrm{E}$ staining (final magnification, $\times 10$ ). Representative images are shown

Skeletal muscle biopsy was performed at age 13 years and 2 months from left quadriceps, after signing informed consent. Routine histological and histochemical techniques, including immunofluorescence study were performed. Haemotoxylin and eosin staining showed slight fibre size variation, absence of degeneration and necrosis, and minimal increase of connective tissue. Immunofluorescence study with specific antibodies for sarcolemmal dystrophin (NCL-DYS1 against Rod domain DYS-ROD, NCL-DYS2 against C-terminal DYS-COOH, NCL-DYS3 against Nterminal DYS-NH, Novocastra, UK) showed normal staining for DYS-NH and DYS-ROD, whereas expression of DYS-COOH, corresponding to the last 17 amino acids, was nearly absent. Other DGC-complex proteins, including beta-dystroglycan (NCL-b-DG Novocastra, UK) and sarcoglycans showed normal staining. Immunofluorescence for utrophin (NCL-DRP2, Novocastra, UK), which is expected increased in DMD patients, did not show an increased expression of the protein (Fig. 1).

Genomic DNA was extracted from peripheral blood leukocytes using standard protocol and $D M D$ gene deletions and duplications were tested by multiplex-ligation-dependent-probe-amplification (MLPA) (SALSA P034 and SALSA P035, MRC-Holland). MLPA analysis surprisingly demonstrated a single deletion of exon 78 in hemizygous (Fig. 2a). Segregation analysis showed that the deletion was not present in the mother, however one older sister was found heterozygous carrier, implying germinal mosaicism in the mother. A 30-year-old brother was also tested for diagnostic and prognostic purposes, but he resulted normal. 
Fig. 2 a Multiplex-ligationdependent-probe-amplification result showing the absence of exon 78 (arrow) in proband, and a reduction of the peak in the sister (arrow head),

corresponding to the carrier status. b cDNA sequencing for exons 77-79 of $D M D$ gene in normal control and in proband. Amino acid sequence is indicated below the electropherogram. Asterisk correspond to stop codon in the correct cDNA sequence. In proband, exon 78 (orange bar) is missing, and a new boundery between exon 77 (yellow bar) and exon 79 (light blue bar) is formed. Exon 79 is 28 aa longer due to the loss of the reading frame and the shift of the stop codon (stop loss). The new amino acid sequence of exon 79 is shown in red. (colour figure online)
A

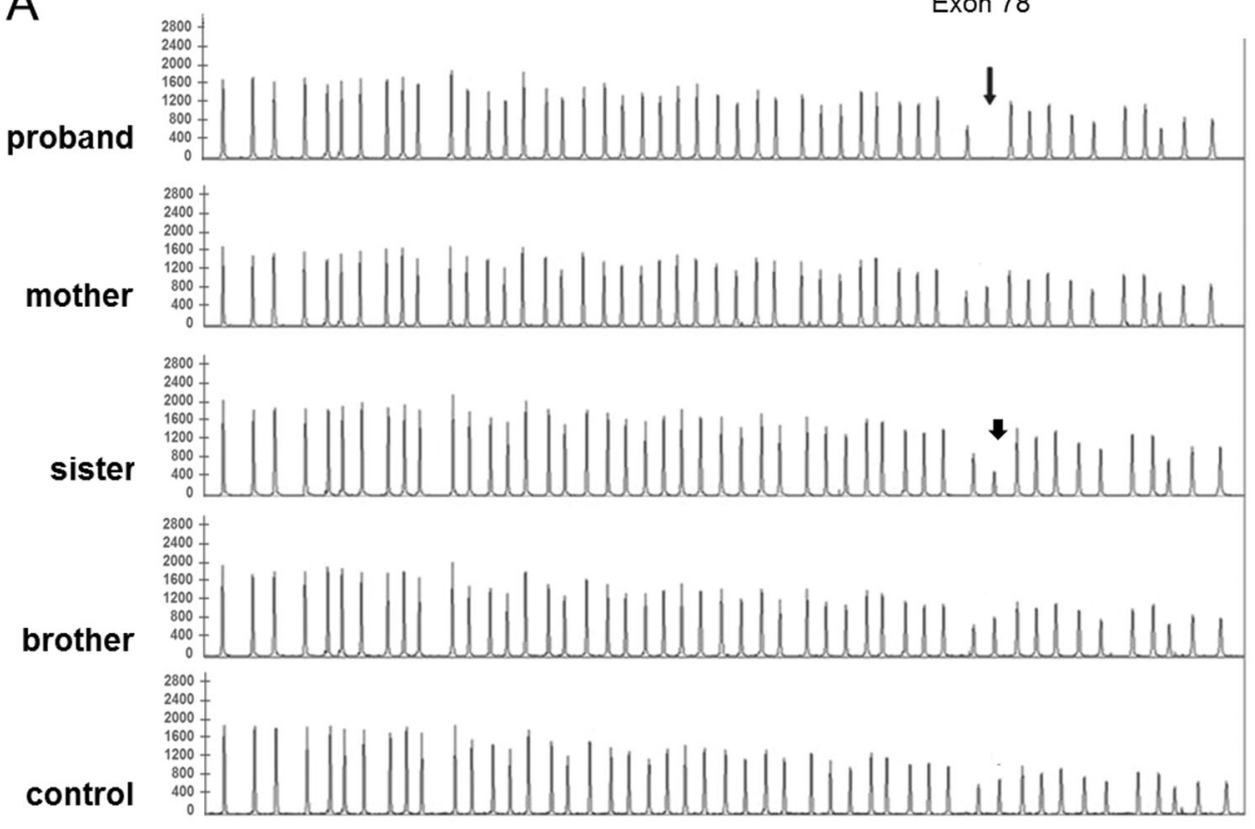

B

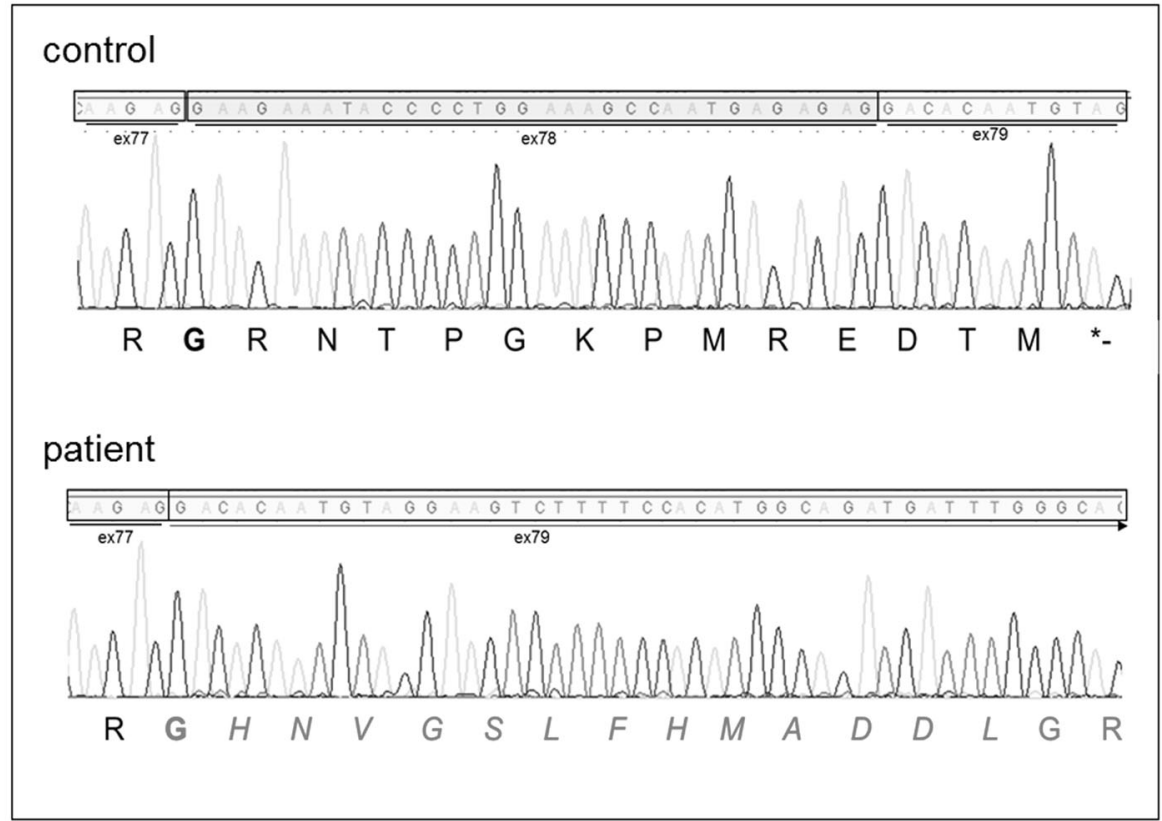

PCR amplification of exon 78 from genomic DNA, with intronic primers $5^{\prime}$-ttttccctttctgatatctctgc-3' and $3^{\prime}$-cetctccacttgcagctcat-5', showed absence of the expected amplificated fragment, confirming the deletion of the exon 78.

To explore the actual effect of the DNA deletion on mRNA, we performed cDNA direct sequencing for exons 77-79 with ad hoc designed primers. Sequences of this portion confirmed the total absence of the $32 \mathrm{bp}$, corresponding to the 11 amino acids of exon 78 (Fig. 2b). Exclusion of exon 78 from $D M D$ transcript changes the ORF of the last exon 79. The new ORF generates a new downstream stop codon, producing a dystrophin protein of 3703 amino acids instead of 3685 amino acids. Bioinfomatic study in ExPASy 2.0 software confirmed the loss of the reading frame and creation of incorrect amino acid sequence in the very last part of the dystrophin. 


\section{Discussion}

In this study, we report on a BMD patient with very mild clinical presentation, carrying a previously unreported deletion of exon 78 in $D M D$ gene.

According to prediction, this deletion should lead to a loss of the reading frame with putative DMD phenotype. Several authors have already delineate exceptions of this reading-frame rule, accounting for up to $10 \%$ of cases $[8,9]$. This patient adds a novel mutation to the list of mutations in $D M D$ gene that do not follow this rule. Furthermore, the effect of specific mutations affecting the C-terminal domain is matter of debate, as they give rise to both severe DMD or unexpectedly mild BMD phenotype [10]. A possible explanation for this clinical variability is the evasion of the nonsense-mediated decay in selected cases [11]. Our case is of particular interest because single-genomic deletion of exon 78 associated to DMD or BMD phenotype has never been object of study in human. Abnormal splicing of $D M D$ exon 78 has been described in dystrophic muscles of patients affected by myotonic dystrophy type 1 , as consequence of the $D M P K$ gene defect [12]. According to authors, aberrant exon 78 deleted $D M D$ compromises muscle fibre maintenance and contributes to the progressive dystrophic process. Conversely, histopathology study of our patient suggests that exon 78 deletion only minimally affects muscle fibres morphology and architecture (Fig. 1f).

We also demonstrated that deletion of exon 78 changes the ORF of exon 79, resulting in a dystrophin protein of 3703 amino acids instead of 3685 amino acids, presumably without significantly impairing its function. In fact, the binding sites for beta-dystroglycan and other proteins of DGC complex reside before exon 78 , in particular, in exons 63-70 for beta-dystroglycan. Accordingly, ImmunoFluorescence studies demonstrated that binding for betadystroglycan is preserved. Thus, we hypothesize that loss of $D M D$ exon 78 does not interfere with formation of normal dystrophin protein and functional DGC complex, whereas the absent expression of C-terminal antibody is only due to technical reason (binding antigen of this antibody in the last 17 aa).

Taken together, we believe these data are useful in clinical practice and in deciphering the results of molecular and histopathological investigations of dystrophinopathy patients.

\section{Compliance with ethical standards}

Conflict of interest The authors declare that they have no conflict of interest.

Ethical publication statement All authors confirm they have read the position on issues involved in ethical publication and affirm that this report is consistent with those guidelines.

\section{References}

1. Worton RG, Thompson MW. Genetics of Duchenne muscular dystrophy. Annu Rev Genet. 1988;22:601-29.

2. Koenig M, Monaco AP, Kunkel LM. The complete sequence of dystrophin predicts a rod-shaped cytoskeletal protein. Cell. 1988;53:219-28.

3. Ahn AH, Kunkel LM. The structural and functional diversity of dystrophin. Nat Genet. 1993;3:283-91.

4. Freund AA, Scola RH, Arndt RC, Lorenzoni PJ, Kay CK, Werneck LC. Duchenne and Becker muscular dystrophy: a molecular and immunohistochemical approach. Arq Neuropsiquiatr. 2007;65:73-6.

5. Koenig M, Beggs AH, Moyer M, Scherpf S, Heindrich K, Bettecken T, et al. The molecular basis for Duchenne versus Becker muscular dystrophy: correlation of severity with type of deletion. Am J Hum Genet. 1989;45:498-506.

6. Den Dunnen JT, Grootscholten PM, Bakker E, Blonden LA, Ginjaar HB, Wapenaar MC, et al. Topography of the Duchenne muscular dystrophy (DMD) gene: FIGE and cDNA analysis of 194 cases reveals 115 deletions and 13 duplications. Am J Hum Genet. 1989;45:835-47.

7. Flanigan KM. Duchenne and Becker muscular dystrophies. Neurol Clin. 2014;32:671-88.

8. Aartsma-Rus A, Van Deutekom JC, Fokkema IF, Van Ommen GJ, Den Dunnen JT. Entries in the Leiden Duchenne muscular dystrophy mutation database: an overview of mutation types and paradoxical cases that confirm the reading-frame rule. Muscle Nerve. 2006;34:135-44.

9. Aartsma-Rus A, GinJaar IB, Bushby K. The importance of genetic diagnosis for Duchenne muscular dystrophy. J Med Genet. 2016;53:145-51.

10. Crawford GE, Faulkner JA, Crosbie RH, Campbell KP, Froehner SC, Chamberlain JS. Assembly of the dystrophin-associated protein complex does not require the dystrophin $\mathrm{COOH}$-terminal domain. J Cell Biol. 2000;150:1399-410.

11. Kerr TP, Sewry CA, Robb SA, Roberts RG. Long mutant dystrophins and variable phenotypes: evasion of nonsense-mediated decay? Hum Genet. 2001;109:402-7.

12. Rau F, Lainé J, Ramanoudjame L, Ferry A, Arandel L, Delalande $\mathrm{O}$, et al. Abnormal splicing switch of DMD's penultimate exon compromises muscle fibre maintenance in myotonic dystrophy. Nat Commun. 2015;6:7205. 